\title{
The Use of Artificial Neural Networks for Differential Diagnosis between Vesicoureteral Reflux and Urinary Tract Infection in Children
}

\author{
(1) Ahmet Keskinoğlu', (1) Su Özgür2 \\ ${ }^{1}$ Ege University Faculty of Medicine, Department of Pediatric Nephrology, Izmir, Turkey \\ 2Ege University Faculty of Medicine, Department of Biostatistics and Medical Informatics, İzmir, Turkey
}

\begin{abstract}
Aim: Vesicoureteral reflux (VUR) and urinary tract infection (UTI) are common problems in children. Our goal is to use different models for the clinical decision of differential diagnosis of VUR and UTI in children.

Materials and Methods: This was a retrospective cross-sectional study with 611 pediatric patients enrolled. Detailed information for the patients was obtained from hospital records and patient files. Three models including different variables were evaluated via an artificial neural network for the differential diagnosis of VUR and recurrent UTI. Clinical findings were included in Model 1, clinical and laboratory findings were included in Model 2, and clinical, laboratory and detailed urinary ultrasonography (USG) findings were included in Model 3. A cross-validation technique was used to evaluate predictive models by partitioning the original sample into a training set to train the model, and a test set to evaluate it.

Results: Of the 611 children, 425 (69.6\%) had VUR and 186 (30.4\%) had UTI. The sensitivity of Model 1 and Model 2 were 0.682 and 0.856 , respectively. Also, Model 3 showed the best performance and highest sensitivity with 0.939 for differential diagnosis.

Conclusion: Differential diagnosis between VUR and UTI in children can be predicted by using clinical, laboratory and USG variables via an Artificial Neural Network. Model 3, which included clinical, laboratory and USG variables together, showed the best performance and highest sensitivity.

Keywords: Artificial neural network, differential diagnosis, urinary tract infection, urinary ultrasonography, vesicoureteral reflux
\end{abstract}

\section{Introduction}

Vesicoureteral reflux (VUR) should be considered in children who have urinary tract anomalies diagnosed by fetal ultrasonography (USC) and who have recurrent urinary tract infection (UTI). For child patients presenting with primary or recurrent UTI, $25-40 \%$ are found to have VUR. However, the actual cause-and-effect relationship between VUR and UTI is controversial (1-3). In a meta-analytic study, it was reported that children with VUR had a higher risk of pyelonephritis and renal scarring than those without VUR (Odds Ratio: 2.8 and 3.8, respectively) (4). The prevalence of VUR in children with end-stage renal failure is $3-25 \%$ (5). Some authors have suggested that renal scarring is not caused by VUR alone, but by concomitant congenital renal hypoplasia or dysplasia (6).

Around one-third of children who were diagnosed with a UTI have VUR. Imaging tests are used for diagnosis in cases where VUR is suspected. Voiding cystourethrogram (VCUG), 
the gold standard radiographic test used to diagnose VUR, is a widely accepted test (7). Screening of VCUG for VUR in siblings and neonates with prenatal hydronephrosis is recommended $(7,8)$.

However, renal/bladder ultrasound (RBUS), which is a noninvasive procedure, is commonly used as an initial screening test to determine VUR. The diagnostic accuracy of RBUS is controversial. Several studies report that the RBUS has low sensitivity and specificity for detecting VUR in children with UTI, and sometimes it may be not possible to detect high-grade VUR. In one study, RBUS sensitivity for high-grade (i.e., Grades IV-V) VUR was found to be $86 \%$, while in another study, RBUS sensitivity and specificity was found to be $40 \%$ and $76 \%$, respectively. At the same time, the diagnostic value of ultrasound with clinical and laboratory findings is not discussed in the mentioned studies above (9-12). Children with low-grade reflux should be followed under observation. Prophylactic antibiotic therapy should be suggested for patients with bladder and bowel dysfunction and for patients who are not toilet trained. Surgical correction is performed in those patients with persistent grade IV or grade $\mathrm{V}$ after they have reached two or three years of age. Conversely, the treatment method in patients with low-grade VUR and renal scarring is controversial.

The presence of renal scarring changes the management of VUR. Early detection of VUR should be performed to prevent the development of renal scarring, and prognosis of the patient should be closely monitored. In addition, the accuracy of early detection and prognostic monitoring methods should be known. In recent studies, some different analytical methods, such as artificial neural network (ANN), have been used to determine the accuracy of diagnostic tests and differential diagnosis with classical approaches. Classical approaches lead to misinterpretations when complex data structures are encountered. Therefore, analytic methods such as ANN, in which multiple data are evaluated together, are important for management (13-15).

In the neural network model, the activation function is defined as the following linear function $f(x)=x$, where $x$ represents a parameter of the activation function. $b$ is called the bias term and it is associated with each inter connection to introduce a supplementary degree of freedom. The weighted sum $S$ to the $i_{\text {th }}$ neuron in the $k_{\text {th }}$ layer $(k \geq 2)$ is

$$
S_{k .1}=\sum_{f_{=1}}^{N_{k=1}}\left[\left(w_{\kappa-1} x_{\kappa-1+f}\right)+b_{\kappa-1}\right]
$$

where $w$ is the weight parameter between each neuron-neuron inter connection (8).

Multiple data structures should be evaluated together in children with VUR for an early differential diagnosis and for the prediction of prognosis (16). In the literature, classical statistical methods such as multivariate regression analysis are frequently used in the prediction of differential diagnosis and prognosis of VUR (17). In these analytic methods where there are multiple linear connections, the co-evaluation of multiple variables has some drawbacks. Strong predictions by ANNs can be made in situations where data structures need to be evaluated together (18). The structure of these networks supports capturing very complex relationships between predictor variables and dependent variables. This study has features that require analysis with ANN. The high number of variables and the possibility of multiple correlations form the basis of different statistical approaches.

In this study, three different ANN models were created in which different clinical, laboratory and imaging variables were included. Thus, the contribution of different findings in these models to the differential diagnosis was evaluated.

\section{Materials and Methods}

This study was approved by the Ethics Committee of the Faculty of Medicine of Ege University (The protocol number: 13-6.1/56 ). Patients gave their informed consent for inclusion in the study.

In this retrospective cross-sectional study, 611 pediatric patients who had been admitted to Ege University Faculty of Medicine Pediatric Nephrology Outpatient Clinic and Tepecik Training and Research Hospital were included. Informative data about the patients were obtained from hospital records and patient files. The conversion of records into data was carried out by pediatric nephrologists in the study team and a database was created. Four basic variables of the patients (gender, age group, history of UTI, and urine culture positive UTI) were examined descriptively. 39 characteristics including these four variables were evaluated by ANN algorithms (Figure 1).

In our study, 39 features (physical findings, laboratory and imaging findings) were presented (Table I). VUR or UTI, which is classified by pediatric nephrologists, constitutes the outcome variable to be estimated. History of UTI and culture positive UTI used to estimate the outcome variable are two independent variables. Since recurrent UTI which occurs commonly in children with VUR is an important variable, history of UTI was included in the 
model. Three different models were created by an ANN method for the differential diagnosis of VUR/UTI. ANN analyses of 14 clinical and 16 laboratory variables without any ultrasonographic variables were included in Model 1. In Model 2, 14 clinical, 16 laboratory and 2 ultrasonographic variables (hydronephrosis and dilatation) were included in the ANN analysis. In Model 3, 14 clinical, 16 laboratory, and 10 ultrasonographic variables ( 9 hydronephrosis and 1 dilatation) were included in the ANN analysis.

All variables were used in the analysis as shown in Table I. Variability of the differences of AP (anteroposterior diameter) was used by re-coding in Model 3 (19).

Eleven children had bilateral VUR. The anteroposterior (AP) renal pelvis diameter of the right and left kidneys were

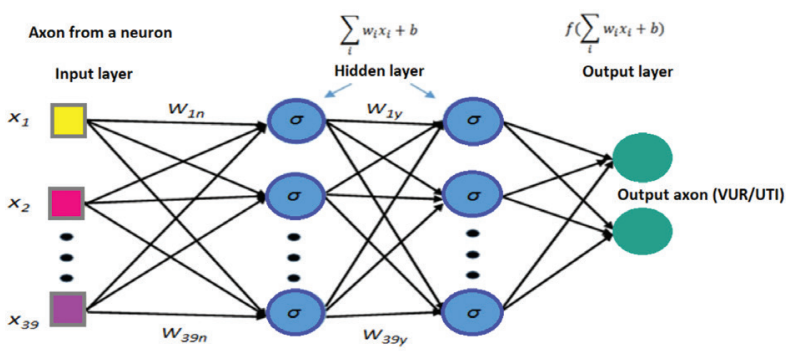

Figure 1. Perceptron network structure with 39-input and 2-output multilayer forward feed

VUR: Vesicoureteral reflux, UTI: Urinary tract infection not included separately in the model as kidney diameters were determined in only 3 children. Values of AP right and left renal diameter difference are defined categorically according to the values of either $\leq 5 \mathrm{~mm}$ or $\geq 5 \mathrm{~mm}$ in analytic methods.

As a result, an axon leading from an ANN, reaching the synapse dendrite (studied with 39 variables) was studied by the linear modeling system and the variable output was predicted as VUR/UTI.

\section{Statistical Analysis}

Statistical analyses were performed using Waikato Environment for Knowledge Analysis (WEKA 3.8).

$\mathrm{K}$-fold cross-validation technique was used to evaluate predictive models by partitioning the original sample into a training set to train the model, and a test set to evaluate it. In 10-fold cross-validation, the original sample was randomly partitioned into 10 equal-sized subsamples. Of the 10 subsamples, a single subsample was retained as the validation data for testing the model, and the remaining 9 subsamples were used as training data. The cross-validation process was then repeated 10 times, with each of the 10 subsamples used exactly once as the validation data. The 10 results were then averaged to produce a single estimation.

The significance of the area under the curve (AUC)

Table I. The variables used in the artificial neural network analysis

\begin{tabular}{|c|c|c|}
\hline Clinical variables & Laboratory variables & USG variables \\
\hline Diagnosis $(\mathrm{V} / \mathrm{I})^{\text {Model 1,2,3 }}$ & u-culture $_{(\mathbf{c})}$ Model 1,2,3 & USG-R-grade $_{\text {(ordinal:0,1,2) }}$ Model 3 \\
\hline Gender $_{\text {(cat: Male/Female) }}$ Model 1,2,3 & ud-density $_{(\mathbf{c})}$ Model 1,2,3 & USG-L-grade ${ }_{\text {(ordinal: } 0,1,2 \text { ) }}^{\text {Model } 3}$ \\
\hline Age $_{\text {(c) }}$ Model 1,2,3 & ud-nitrite $_{(\text {cat:Y/N) }}$ Model 1,2,3 & USG-AP diameter differences ${ }_{(\mathrm{cat} \leq 5,5<)^{\text {Model3 }}}$ \\
\hline Fever $_{\text {(cat:Y/N) }}$ Model 1,2,3 & ud-l.esterase $_{(\text {cat:Y/N) }}$ Model 1,2,3 & USG-R-Hydronephrosis (cat:Y/N) $^{\text {Model 2, } 3}$ \\
\hline Emesis $_{(\text {catty/N) }}$ Model 1,2,3 & ud-erythrocyte $_{(\text {cat:Y/N) }}$ Model 1,2,3 & USG-L-Hydronephrosis (cat:Y/N) \\
\hline Incontinence $_{(\text {cat:Y/N) }}$ Model 1,2,3 & ud-protein $_{(\text {cat:Y/N) }}$ Model 1,2,3 & USG-R-L Hydronephrosis (cat:Y/N) $^{\text {Model 2, } 3}$ \\
\hline Collywobbles $_{(\text {cat:Y/N) }}$ Model 1,2,3 & us-erythrocyte $_{\text {(cat:Y/N) }}$ Model 1,2,3 & USG-Bladder wall thickening ${ }_{(\text {cat:Y/N) }}$ Model 3 \\
\hline Urgency $_{(\text {cat:Y/N) }}$ Model 1,2,3 & us-leukocyte $_{(\text {cat:Y/N) }}$ Model 1,2,3 & USG-Bladder diverticulum ${ }_{(\text {cat:Y/N) }}$ Model 3 \\
\hline Frequent urination ${ }_{(\text {cat:Y/N) }}$ Model 1,2,3 & ud-leukocyte $_{\text {(cat:Y/N) }}$ Model 1,2,3 & USG-Ureter dilatation ${ }_{(\text {cat:Y/N) }}$ Model 3 \\
\hline Dysuria $_{(\text {cat:Y/N) }}$ Model 1,2,3 & us-bacteria $_{\text {(cat: } \mathrm{Y} / \mathrm{N})}$ Model 1,2,3 & \\
\hline Restless $_{(\text {cat:Y/N) }}$ Model 1,2,3 & us-leukocyte cylinder ${ }_{(\mathbf{c})}$ Model 1,2,3 & \\
\hline Anorexia $_{(\text {cat:Y/N) }}$ Model 1,2,3 & b-leukocyte ${ }_{(c)}^{\text {Model 1,2,3 }}$ & \\
\hline UTI on story $_{(\text {cat:Y/N) }}$ Model 1,2,3 & b-thrombocyte $_{\text {(c) }}$ Model 1,2,3 & \\
\hline \multirow[t]{3}{*}{ Prolonged neonatal jaundice $_{(\text {cat:Y/N) }}$ Model 1,2,3 } & b-urea $_{(c)}$ Model 1,2,3 & \\
\hline & b-creatinine $_{(c)}$ Model 1,2,3 & \\
\hline & b-uric acid ${ }_{(\mathbf{c})}$ Model $1,2,3$ & \\
\hline
\end{tabular}


statistics was assessed by receiver operating characteristic (ROC) analysis. The statistics (sensitivity, specificity and precision value) obtained from the ROC analysis were also evaluated.

\section{Results}

Of the 611 children, 425 (69.6\%) had VUR and 186 (30.4\%) had UTI. Some descriptive characteristics of the children with VUR and UTI are presented in Table II.

$41.2 \%$ (175) of the children with VUR were boys and $58.8 \%$ (250) were girls. $41.9 \%$ (78) of the children with UTI were boys and $58.1 \%$ (108) were girls. $55.1 \%$ of those children with VUR and $58.1 \%$ of those children with recurrent UTI were in the age group of 0-24 months. During medical examination, $76.5 \%$ of children with VUR and $78.9 \%$ with UTI presented their urinary cultures. The respective numbers of children with urinary culture were similar for both VUR and recurrent UTI. Since VUR data was related with UTI variable, it is hard

Table II. Characteristics of children with vesicoureteral reflux and urinary tract infection

\begin{tabular}{|c|c|c|c|c|}
\hline \multirow{2}{*}{ Variables } & \multicolumn{2}{|l|}{ VUR } & \multicolumn{2}{|l|}{ UTI } \\
\hline & n (425) & $\%$ & n (186) & $\%$ \\
\hline \multicolumn{5}{|l|}{ Gender } \\
\hline Boy & 175 & 41.2 & 78 & 41.9 \\
\hline Girl & 250 & 58.8 & 108 & 58.1 \\
\hline
\end{tabular}

\begin{tabular}{|l|l|l|l|l|}
\hline Age groups \\
\hline 0-24 months & 234 & 55.1 & 108 & 58.1 \\
\hline $25-60$ months & 83 & 19.5 & 34 & 18.3 \\
\hline 61 months and over & 108 & 25.4 & 44 & 23.7 \\
\hline
\end{tabular}

\section{Presence of urinary culture in previous UTI}

\begin{tabular}{|l|l|l|l|l|}
\hline Present & 315 & 76.5 & 146 & 78.9 \\
\hline Not present & 97 & 23.5 & 39 & 21.1 \\
\hline UTI based on notification & 101 & 23.8 & 31 & 16.7 \\
\hline Not present & 259 & 60.9 & 118 & 63.4 \\
\hline $1-4$ & 65 & 15.3 & 37 & 19.9 \\
\hline 5 and over & VUR: Vesicoureteral reflux, UTI: Urinary tract infection \\
\hline
\end{tabular}

Table III. Results of model 1, 2 and 3

\begin{tabular}{|l|l|l|l|l|}
\hline Model & Sensitivity & Specificity & Precision & AUC \\
\hline Model 3 & 0.939 & 0.418 & 0.779 & 0.809 \\
\hline Model 2 & 0.856 & 0.425 & 0.744 & 0.747 \\
\hline Model 1 & 0.682 & 0.216 & 0.527 & 0.601 \\
\hline \multicolumn{5}{|l}{ AUC: Area under the curve } \\
\hline
\end{tabular}

to differentiate the diagnosis of VUR and UTI. The results of ANN for VUR and UTI are shown in Table III.

By the cross-validation method, the sensitivity to differentiate VUR in Model 3 was found to be 0.939 whereas the specificity was 0.418. Also, AUC was found to be a significantly high value at 0.809 . Model 2 included 2 ultrasonographic variables (hydronephrosis and dilatation). The sensitivity of model 2 was found to be 0.856 and AUC was found to be 0.747. In Model 1, in which there was no ultrasonographic variable included, the sensitivity was found to be 0.682 and AUC was found to be 0.601. The ROC which was obtained from ANN analysis is presented Figure

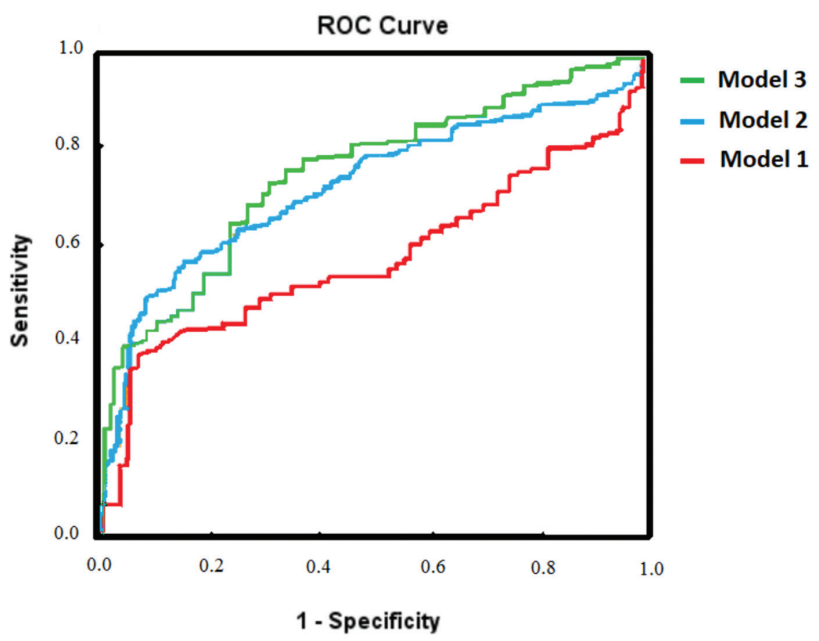

Figure 2. Comparisons of the model 1 , model 2 , model 3 ROC: Receiver operating characteristic

2. When the AUC's were evaluated for $A N N$, it was found that the RBUS variable is very important in differentiating VUR and UTI.

\section{Discussion}

In this study, the performances of 3 different models were evaluated via ANN analysis for the differential diagnosis of VUR and recurrent UTI, which is an important urinary problem in children. Different sensitivity, specificity and predictive values were obtained by using different models that incorporate different findings. When detailed RBUS findings are added to the models, the sensitivity value increased from 0.682 to 0.939 , while specificities were similar. However, the predictive value of Model 3 was higher than the other models because the differential diagnosis of VUR was the main objective. In the model that includes most variables, clinical, laboratory and detailed urinal tract findings (10 clinical variables, 14 laboratory variables, and 
10 ultrasonographic variables) were used together and the predictive value and sensitivity were determined to be 0.779 and 0.939 , respectively. In the literature, lower sensitivity and lower specificity estimates of RBUS in the detection of VUR have been reported. However, the findings of RBUS have not been detailed in these studies.

In one study, the sensitivity of colored doppler RBUS in the evaluation of ureteral jet opening in children with VUR was determined to be $85 \%$ for grade III-IV and $94 \%$ for grade IV-V (20).

The sensitivity of RBUS was found to be 0.939 in our study. We think that the diagnostic sensitivity of VUR will increase when RBUS with a discriminative capability such as color doppler RBUS is used together with conventional RBUS.

Some studies reported that ANN and other data mining methods supported medical decisions regarding VUR and some nephrological problems (21-27).

In one study, data was evaluated with ANN, and the results were compared with logistic regression analysis results for the surgical treatment decision of VUR (24). Better performance has been achieved with ANN.

\section{Study Limitations}

In our study, the performance of ANN was tested and found to be distinctive in a more chaotic decision, such as the differential diagnosis between VUR and UTI. We found that the model with the highest distinguishing characteristics was the model including the detailed RBUS variables.

The algorithms were trained on the training set via a multilayer perceptron before the estimates were made on the test set (28). The iterations were continued until the error values between the input and output parts of the algorithm were minimized. Algorithms which were obtained via $\mathrm{k}$-fold cross validation were applied on the validation set.

It is important to select the ANN function according to the suitability of the data structure. A linear ANN algorithm was used in our study. Since this approach involves the training stages of the algorithms, more successful estimates can be obtained than with classical linear approaches (29). When the outputs of our study are evaluated together with the literature, detailed clinical findings and detailed ultrasonographic evaluation were observed to be important for the differential diagnosis between VUR and UTI. For this reason, the importance of RBUS, which is frequently performed in renal disease, can be seen and so negating the need for invasive methods. Results with high sensitivity were obtained by evaluating multiple data using different analysis techniques such as ANN. Some studies also have reported that better estimates were obtained by the ANN method.

\section{Conclusions}

VUR is a urinary system anomaly that causes adverse outcomes in children such as renal scarring when late diagnosis or uncontrolled prognosis occurs. Early diagnosis and differential diagnosis from UTI are important. ANNs can build computer models that are useful for medical decisionmaking. When the data from detailed clinical findings and noninvasive imaging techniques such as USC are evaluated together via ANN analysis, better estimates and higher sensitivity can be obtained for the differential diagnosis of VUR and UTI.

\section{Acknowledgements}

This article was written based on a project supported by TUBITAK with project number 114 S011.

We would like to thank Assoc. Prof. Nejat Aksu who from Tepecik Training and Research Hospital Department of Pediatric Health and Diseases for their support and contributions to our research. Dr. Nejat Aksu contributed to the conception and design of this study, the acquisition, analysis and interpretation of data and results and the writing of the manuscript. Unfortunately, we lost him. We commemorate him with respect.

\section{Ethics}

Ethics Committee Approval: The study was approved by the ethics committee of the Ege University Faculty of Medicine the protocol number: 13-6.1/56 the date of approval: 29.07.2013).

Informed Consent: Patients have given informed consent for participation in the study.

Peer-review: Externally peer-reviewed.

\section{Authorship Contributions}

Concept: A.K., S.Ö., Design: A.K., S.Ö., Data Collection or Processing: A.K., S.Ö., Analysis or Interpretation: A.K., S.Ö., Writing: A.K., S.Ö.

Conflict of Interest: The authors declared no potential conflicts of interest with respect to the research, authorship, and publication of this article.

Financial Disclosure: The authors declared that this study received no financial support. 


\section{References}

1. Blumenthal I. Vesicoureteral reflux and urinary tract infection in children. Postgrad Med J 2006; 82:31-5.

2. Swerkersson S, Jodal U, Sixt R, Stokland E, Hansson S. Relationship among vesicoureteral reflux, urinary tract infection and renal damage in children. J Urol 2007; 178:647-51.

3. Routh JC, Bogaert GA, Kaefer $M$, et al. Vesicoureteral reflux: current trends in diagnosis, screening, and treatment. Eur Urol 2012; 61:773-82.

4. Faust WC, Diaz M, Pohl HG. Incidence of post-pyelonephritic renal scarring: a meta-analysis of the dimercapto-succinic acid literature. I Urol 2009; 181:290-7.

5. Brakeman P. Vesicoureteral reflux, reflux nephropathy, and endstage renal disease. Adv Urol 2008; 508949

6. Hinchliffe SA, Chan YF, Jones $\mathrm{H}$, Chan N, Kreczy A, van Velzen D. Renal hypoplasia and postnatally acquired cortical loss in children with vesicoureteral reflux. Pediatr Nephrol 1992; 6:43944.

7. Management and screening of primary vesicoureteral reflux in children: AUA Guideline (2010), 2017.

8. Sunaryo PL, Cambareri GM, Winston DG, Hanna MK, Stock JA. Vesico-ureteric reflux (VUR) management and screening patterns: are paediatric urologists following the 2010 American Urological Association (AUA) guidelines? BJU Int 2014; 114:761-9.

9. Alshamsam L, Al Harbi A, Fakeeh K, Al Banyan E. The value of renal ultrasound in children with a first episode of urinary tract infection. Ann Saudi Med 2009; 29:46-9.

10. Sorkhi H, Nooreddini HG, Amiri M, Osia S, Farhadi-Niakee S. Prediction of vesicoureteral reflux in children with first urinary tract infection by dimercaptosuccinic Acid and ultrasonography. Iran J Pediatr 2012; 22:57-62.

11. Lee HY, Soh BH, Hong CH, Kim MJ, Han SW. The efficacy of ultrasound and dimercaptosuccinic acid scan in predicting vesicoureteral reflux in children below the age of 2 years with their first febrile urinary tract infection Pediatr Nephrol 2009, 24:2009-13.

12. Mahant S, Friedman J, MacArthur C. Renal ultrasound findings and vesicoureteral reflux in children hospitalised with urinary tract infection Arch Dis Child 2002; 86:419-20.

13. Chapoy A, Mohammadi AH, Richon D. Predicting the hydrate stability zones of natural gases using artificial neural networks. Oil Gas Sci Technol 2007; 62:701-6.

14. Emoto $T$, Abeyratne UR, Chen $Y$, Kawata I, Akutagawa $M$, Kinouchi Y. Artificial neural networks for breathing and snoring episode detection in sleep sounds Physiol Meas 2012; 33:167589.
15. Hu K, Wan JQ, Ma YW, Wang Y, Huang MZ. A fuzzy neural network model for monitoring $A 2 / O$ process using on-line monitoring parameters. J Environ Sci Health A Tox Hazard Subst Environ Eng 2012; 47:744-54.

16. Seckiner I, Seckiner SU, Erturhan S, Erbagci A, Solakhan M, Yagci F. The use of artificial neural networks in decision support in vesicoureteral reflux treatment. Urol Int 2008; 80:283-6.

17. Knudson MJ, Austin JC, Wald M, Makhlouf AA, Niederberger CS, Cooper CS. Computational model for predicting the chance of early resolution in children with vesicoureteral reflux. I Urol 2007; 178:1824-7.

18. Maind SB, Wankar P. Research paper on basic of artificial neural network. Int I Rec Innov Trends Comput Communicat 2014; 2:96-100

19. Nguyen HT, Herndon CD, Cooper C, et al. The Society for Fetal Urology consensus statement on the evaluation and management of antenatal hydronephrosis. I Pediatr Urol 2010; 6:212-31.

20. Asanuma $\mathrm{H}$, Matsui $\mathrm{Z}$, Satoh $\mathrm{H}$, et al. Color doppler ultrasound evaluation of ureteral jet angle to detect vesicoureteral reflux in children. J Urol 2016; 195:1877-82

21. Shoskes D, Ty R, Barba L, Sender M. Prediction of the early graft function in renal transplantation using a computer neural network. Transplant Proc 1998; 30:1316-7.

22. Finne $\mathrm{P}$, Finne $\mathrm{R}$, Stenman UH. Neural network analysis of clinicopathological factors in urological disease: a critical evaluation of available techniques. BJU Int 2001; 88:825-31.

23. Serrano-Durbá A, Serrano AJ, Magdalena IR, et al. The use of neural networks for predicting the result of endoscopic treatment for vesico-ureteric reflux. BJU Int 2004; 94:120-2.

24. Ducher M, Kalbacher E, Combarnous F, et al. Comparison of a bayesian network with a logistic regression model to forecast IgA nephropathy. Biomed Res Int 2013; 686150.

25. Liu X, Pei X, Li N, et al. Improved glomerular filtration rate estimation by an artificial neural network. PLOS ONE 2013; 8:58242.

26. Lu TC, Tseng CY. Hemodialysis key features mining and patients clustering technologies. Adv Artificial Neural Systems, 2012.

27. Douali N, Papageorgiou El, De Roo J, Cools H, Jaulent MC. Clinical decision support system based on fuzzy cognitive maps. J Comput Sci Syst Biol 2015; 8:112-20.

28. Artificial Neural Networks - Methodological Advances and Biomedical Applications. Suzuki K ed, InTech, 2011.

29. Dayhoff JE, DeLeo JM. Artificial Neural Networks. Cancer 2001 91(Suppl S8):1615-35. 